\title{
Visual Search in ASD: Instructed Versus Spontaneous Local and Global Processing
}

\author{
Ruth Van der Hallen ${ }^{1,2,3} \cdot$ Kris Evers $^{1,2,3,4} \cdot$ Bart Boets $^{2,3} \cdot$ Jean Steyaert $^{2,3}$. \\ Ilse Noens ${ }^{3,4} \cdot$ Johan Wagemans ${ }^{1,3}$
}

Published online: 22 June 2016

(c) Springer Science+Business Media New York 2016

\begin{abstract}
Visual search has been used extensively to investigate differences in mid-level visual processing between individuals with ASD and TD individuals. The current study employed two visual search paradigms with Gaborized stimuli to assess the impact of task distractors (Experiment 1) and task instruction (Experiment 2) on local-global visual processing in ASD versus TD children. Experiment 1 revealed both groups to be equally sensitive to the absence or presence of a distractor, regardless of the type of target or type of distractor. Experiment 2 revealed a differential effect of task instruction for ASD compared to $\mathrm{TD}$, regardless of the type of target. Taken together, these results stress the importance of task factors in the study of local-global visual processing in ASD.
\end{abstract}

Keywords Autism spectrum disorder (ASD) - Visual search · Local-global · Interference · Task instruction . Gabor

Electronic supplementary material The online version of this article (doi:10.1007/s10803-016-2826-1) contains supplementary material, which is available to authorized users.

Ruth Van der Hallen

ruth.vanderhallen@kuleuven.be

1 Laboratory of Experimental Psychology, Department of Brain and Cognition, KU Leuven, 3000 Leuven, Belgium

2 Child and Adolescent Psychiatry, Department of Neurosciences, UPC-KU Leuven, 3000 Leuven, Belgium

3 Leuven Autism Research (LAuRes), KU Leuven, 3000 Leuven, Belgium

4 Parenting and Special Education Research Unit, KU Leuven, 3000 Leuven, Belgium

\section{Introduction}

Visual search, or the ability to locate an object amongst an array of stimuli, has been extensively studied over the past 40 years (Eckstein 2011). The task typically involves an active scan of the visual environment to locate a particular object or feature (the target) among other objects or features (the distractors). Examples of this can be seen in everyday life, for instance, when one is trying to find a product on the shelves of the refrigerator or when one is trying to find a friend in a crowd of people. There are two types of search: feature search and conjunction search. Feature search (also known as "disjunctive" or "efficient" search) is present in cases where the target and the distractors are differentiated by a single property such as color, shape, orientation, or size (Treisman and Gelade 1980). Typical examples are a red X among blue X's, or a green T amongst green Q's. Single feature search is characterized by a "pop-out" effect: the target leaps from the display without any effort on the part of the observer and the search time is unaffected by the set size of the distractors (Wang et al. 1994). Conjunction search (also known as "inefficient" search) occurs when the target and the distractors share similarities in more than one single visual property, and when the combination or the unique conjunction of these properties makes the target stand out (Treisman and Gelade 1980). A typical example is a red X amongst red T's and blue X's. The fact that the target shares a feature with each of the distractors makes the target harder to be identified. This type of search therefore typically requires a more or less serial search strategy. Accordingly, the target no longer pops out and search times vary almost linearly with the number of distractors.

Visual search comprises a set of complex behaviors that encompass many aspects central to cognitive functioning. 
Amongst others, it involves covert visual attention (including zooming, selection, shifting), temporal integration of information across eye movements, memory for configurations, decision strategies, and oculomotor control (Eckstein 2011). The strength of the search paradigm lies in its versatility and the extent to which it can be modified to investigate a wide variety of aspects of visual cognition (e.g. perceptual grouping, working memory, spatial resolution, dual-task interference; for an overview, see Eckstein 2011). In addition, visual search has been used to investigate individual differences, as well as differences between typically developing (TD) groups and specific population groups, such as young children or aging adults, or clinical populations, such as individuals with attention deficit hyperactivity disorder (ADHD) or autism spectrum disorder (ASD).

Along those lines, visual search has been used in particular to investigate differences in mid-level visual processing (e.g., enhanced discrimination or reduced grouping) between individuals with and without ASD. This line of research situates itself within a vast and broader research field on atypical perceptual organization in ASD. Here, the focus lies on the interplay of local and global perception in ASD, and the extent to which that interplay deviates from typical development. Overall, however, research on local and global perception in ASD has yielded inconsistent and contradictory results (for reviews, see Behrmann et al. 2006; Simmons et al. 2009). Although some researchers have found visual atypicalities for ASD in terms of enhanced local perception and others in terms of a diminished performance for global perception, many have failed to replicate one or both of these phenomena. A recent meta-analysis (Van der Hallen et al. 2015a) bundled 56 studies on atypical perceptual organization in ASD and suggests that differences between individuals with ASD and TD individuals are limited to the speed with which global information is processed: Individuals with ASD proved slower in global-order perception than TD individuals, in particular when having to attend to global order while incongruent information is present at the local level. No other consistent group differences were revealed.

However, similar to the larger research field this research is embedded in, research on visual search in ASD has largely yielded inconsistent results. While some researchers found evidence of superior search in autism (e.g., Joseph et al. 2009; O'Riordan et al. 2001), others failed to find any differences (e.g., Iarocci and Armstrong 2014). Instances of superior search in ASD are usually taken as evidence of reduced global visual processing, or increased tendencies or abilities for more local, detail-oriented information processing in ASD (Happé and Booth 2008; Kaldy et al. 2013; Mottron et al. 2006). While the meta-analysis by Van der Hallen et al. (2015a) failed to find a reliable group difference between individuals with ASD and TD individuals for visual search, Kaldy et al. (2013) argue that, based on a mini-review on search in ASD they have conducted, an ASD advantage in visual search does stand on firm empirical ground.

Despite these mixed findings and mixed summary conclusions, the versatility of search paradigms in general, continues to make these paradigms valuable for ASD research. In the current study we pursued this approach and investigated the impact of task distractors (Experiment 1) and the effect of presentation mode and task instruction (Experiment 2) on search in ASD versus TD individuals. Both experiments use a common search paradigm and a similar type of display comprised of Gabor elements. Gabor elements have clearly defined local features such as orientation, spatial frequency and contrast, without being strictly bounded in space and discrete, like line segments. Technically, they are the product of a sinusoidal luminance grating modulated by a two-dimensional Gaussian window. Gabor elements closely model the receptive field properties of orientation-selective simple cells in the primary visual cortex (V1). As they model the known spatial-frequency processing properties of cells in area $\mathrm{V} 1$, the use of these has clear advantages compared to stimuli with unknown effects on V1 neurons (e.g., arbitrarily constructed lines and dots). Displays with Gabor elements are highly controllable and allow for well-controlled experimental designs. For that reason, they have been widely used in low-level vision research and contour integration studies (for a review, see Hess et al. 2015).

In the present studies, embedded in a larger field of background Gabor elements, search targets are defined by a local difference in contrast or orientation, or by a more global arrangement of the Gabor elements (a closed shape or a curvilinear group called a "snake").

In Experiment 1 Gaborized visual search is employed to investigate the impact of interfering distractors. Studying distractor interference in ASD is a relatively new topic. In these types of studies participants are asked to ignore competing distractors while attempting to focus on the target stimulus. For the most part, studies on distractor interference show evidence of increased interference for individuals with ASD, compared to typically developing participants (Adams and Jarrold 2012). This means that, compared to TD individuals, individuals with ASD experience more hindrance of distractors and find it harder to ignore them altogether. Most of the work on distractor interference has been done using classic response inhibition paradigms, such as the flanker paradigm. In a flanker task the target is usually flanked by non-target stimuli which correspond either to the same directional response as the target (congruent flankers), to the opposite response (incongruent flankers), or to neither responses (neutral 
flankers) (Eriksen and Eriksen 1974). Some of the evidence on distractor interference comes from other experimental paradigms, such as the well-known Navon paradigm (Navon 1977). In a standard selective attention version of this paradigm, participants are asked to focus on one level of the stimuli (i.e., the small letters or figures) for one part, and then asked to focus on the other level of the stimuli (i.e., the large letters or figures) for the other part of the experiment. From these studies, one can extract to what extent participants experience interference between levels, and in what direction, small-to-large or large-to-small, this interference is strongest. Here, researchers sometimes find local-to-global interference for ASD, rather than the more common global-to-local interference effect often found in TD (e.g., Wang et al. 2007). Such evidence does not suggest a difference in overall sensitivity to distractors between individuals with and without ASD, but a difference in sensitivity that is dependent on the particular type of distractor and its relationship to the target.

To evaluate these effects of distractor interference further, Experiment 1 entails a Gaborized visual search task where we investigated participants' performance for locating a predefined target and ignoring the distractors, for both local and global targets, and local and global distractors. We hypothesize that children with ASD would experience more local-to-global interference (distractor interference by local distractors), compared to more globalto-local interference (distractor interference by global distractors) for TD individuals.

In Experiment 2 Gaborized visual search is employed to investigate the influence of implicit versus explicit task instructions on local-global visual processing. Real-life situations require implicit and automatic processing, rather than explicit processing. For instance, when driving a car, one needs to be able to evaluate traffic situations on the spot and as an integrated whole in order to react adequately to specific elements in the context of that scene (e.g., a child on the sidewalk, ready to step onto the street). If there is slower integration of the different elements and less automatic global visual processing, as has been suggested for individuals with ASD (Van der Hallen et al. 2015a), one will not be able to respond as adequately and will end up at risk for car accidents or traffic violations. While in real-life situations one is not explicitly informed as to how one should process the incoming information best, experimental test situations often make this explicit, which might help individuals with ASD to adopt the right processing style, thereby masking evidence of possible habitual differences (i.e., a perceptual bias). This difference between the real-life world and an experimental setting might be one of the reasons that visual atypicalities in natural situations are often reported by (family members of) individuals with ASD, but remain difficult to elucidate in experimental settings.
One study that investigated the impact of spontaneous versus instructed visual processing is a study by Koldewyn et al. (2013). Koldewyn and colleagues tested a large group of children on both a free-choice task and an instructed task using Navon stimuli. Although children with ASD showed a reduced preference to report global properties of a stimulus when given a choice, their ability to process global properties when explicitly instructed to do so, was unimpaired. With this research, Koldewyn et al. indicated that children with ASD have intact global information processing abilities, yet are less inclined to attend to and report global information. This study was one of the first to actually hint at the importance of presentation mode for individuals with ASD. Experiment 2 aims to investigate this effect further. Therefore, we administered a Gaborized visual search task in which the implicit or explicit nature of the local versus global task instruction was directly manipulated. Note that implicit versus explicit here refers to the degree to which participants were made aware what particular targets to look for, rather than that they were unaware of the task purpose in general, as is often the case in tasks referred to as 'implicit' tasks. We hypothesize that children with ASD would show intact local and global visual processing in the explicit task conditions, with clearcut instructions, but impaired global visual processing in the implicit task conditions.

\section{Experiment 1}

\section{Methods}

\section{Participants}

The research protocol was administered to 6-to-10-year old Dutch-speaking children who reported normal or corrected-to-normal vision. Demographic details of the ASD and the TD group can be found in Table 1.

The experimental group consisted of 21 children with a formal clinical diagnosis of ASD, diagnosed according to DSM-IV-TR criteria (American Psychiatric Association 2000) in a multidisciplinary team or by a child psychiatrist. Recruitment was set up via the Autism Expertise Centre of the University Hospitals Leuven $(n=13)$ or special needs schools for children with ASD $(n=8)$. ASD diagnoses were re-confirmed within the research protocol using the Dutch version of the Autism Diagnostic Observation Scale conducted by a trained clinical psychologist (ADOS-2: Gotham et al. 2006) in 19 of the 21 children.

The comparison group consisted of 40 TD children, recruited via mainstream schools. Children with a known child psychiatric disorder or children with a first-degree family member with a developmental disorder (information 
Table 1 Experiment 1: participant characteristics (main analysis)

\begin{tabular}{|c|c|c|c|c|c|}
\hline & \multicolumn{2}{|c|}{ ASD group ( $n=21: 18$ boys, 3 girls) } & \multicolumn{2}{|c|}{ TD group ( $n=33: 29$ boys, 4 girls) } & \multirow{2}{*}{$\begin{array}{l}\text { Two-sided } t \text { test } \\
p \text { value }\end{array}$} \\
\hline & M & SD & M & SD & \\
\hline Age (years) & 8 & 1 & 8 & 1 & .66 \\
\hline Verbal IQ & 102 & 13 & 108 & 41 & .11 \\
\hline Performance IQ & 104 & 14 & 105 & 38 & .80 \\
\hline SRS & 92 & 19 & 32 & 22 & $<.0001$ \\
\hline ADOS & 5.43 & 1.83 & N/A & N/A & N/A \\
\hline
\end{tabular}

SRS data of one participant with ASD and of five TD participants are missing

gathered from parents) were excluded. Based on the particular research questions (see Data-Analysis and Results sections), we selected different sub-samples of ASD and TD participants, which were group-wise matched based on intelligence, age and gender-ratio (see Table 1; Tables 4, 5 in Online Appendix 1).

Intellectual abilities were estimated by an abbreviated version (Sattler 2001) of the Wechsler Intelligence Scale for Children, Third Edition (WISC-III-NL; Wechsler 1992). ASD symptomatology was evaluated using the SRS (Roeyers et al. 2011).

\section{Procedure}

The study was approved by the ethical committee of the university hospital, and was incorporated within a larger series of studies on visual perception and socio-emotional processing in ASD. The order of the different experiments was randomized, with long breaks in-between experiments. Participation was rewarded with a small present and parents received a mileage allowance, when applicable.

All participants were individually tested in a quiet and darkened room. Participants were seated approximately $57 \mathrm{~cm}$ from a 17-in. computer screen (pixel resolution 1024 by 768, 16-bit color, $75 \mathrm{~Hz}$ refresh rate). They were presented with a Gaborized search task and were instructed to indicate whether the predefined target was situated on the left or right side of the screen, by pressing one of two keyboard buttons. Participants were encouraged to respond as fast and accurately as possible. The number of participants assigned to each condition can be found in Table 2 .

The experiment consisted of two blocks of 60 trials with an explicit instruction defining the target (local vs. global). In each trial a target stimulus was present. Half of the participants started with the block with the local target and then completed the block with the global target. The other half of the participants started with the block with the global target and then completed the block with the local target (see Table 2). In two-thirds of the trials a distractor was present. If a distractor was present, it was either
Table 2 Number of participants per global type $\times$ block order

\begin{tabular}{llllll}
\hline Block order & ASD & & & TD & \\
\cline { 2 - 3 } & $\begin{array}{l}\text { Shape } \\
(n=9)\end{array}$ & $\begin{array}{l}\text { Snake } \\
(n=12)\end{array}$ & $\begin{array}{l}\text { Shape } \\
(n=23)\end{array}$ & $\begin{array}{l}\text { Snake } \\
(n=17)\end{array}$ \\
\hline Local then global & $n=5$ & $n=6$ & & $n=11$ & $n=9$ \\
Global then local & $n=4$ & $n=6$ & & $n=12$ & $n=8$ \\
\hline
\end{tabular}

present in the same half ( 40 trials) or in the other half (40 trials) of the screen displaying the target. Based on the location of the distractor with regard to the location of the target, we refer to the distractor as "consistent" (same side) or "inconsistent" (other side). At the start of each trial, a two-second fixation cross was followed by the appearance of the test stimulus. The stimulus remained on the screen until the participants had responded. No feedback was provided. All participants completed an extensive step-bystep practice protocol with 6 practice trials before commencing with each block of test trials. Stimuli were presented and responses were recorded within the E-prime environment (E-prime version 1.1.41. PST Inc.).

\section{Stimuli}

Stimuli consisted of $19.79^{\circ}$ by $19.61^{\circ}$ displays of randomly oriented Gabor elements containing a target either on the left or right side of the screen. All stimuli were created with the Grouping Elements Rendering Toolbox (GERT: Demeyer and Machilsen 2012), which is especially wellequipped to generate stimulus displays where a contour is embedded in a field of randomly positioned background elements, and takes into account local density cues that could influence detection of the target (Machilsen et al. 2015).

The local targets were constructed as one single Gabor element displaying a higher contrast than the background Gabor elements. The global targets were constructed from multiple Gabor elements constituting either an open 
curvilinear arrangement (referred to as "a snake" and comprised of approx. 11 Gabor elements) or a closed random shape (a "radial frequency pattern" or RFP, generated by a quasi-random combination of sinusoïdally modulated protrusions and indentations, comprised of approx. 28 Gabor elements). Half of the participants were presented with the snake, the other half were presented RFPs as global pattern (see Table 2).

In the baseline condition, no distractor was present (i.e. none of the background elements differed from the others in terms of contrast, and their orientations were all random without grouping). In the distractor conditions, an additional distinctive element or arrangement was present, which could attract attention in addition to the actual target and could therefore be "distracting". In the consistent distractor condition, the distractor was located in the same half of the screen as the target. In the inconsistent distractor condition, the distractor was located in the opposite half of the screen as the target. In the "local blocks" (contrastdefined single target), distractors consisted of more globally defined groups of elements (snakes or shapes), while in the "global blocks" (where targets were snakes or shapes), distractors were locally distinctive elements (with enhanced contrast). Examples of the stimuli can be found in Fig. 1.

\section{Data-Analysis}

Reaction times (RT) and mean accuracy were analyzed. Only RTs (expressed in milliseconds) of correctly responded trials were analyzed, and RTs smaller than $100 \mathrm{~ms}$ or over $10,000 \mathrm{~ms}$ were excluded from further analysis, resulting in the exclusion of $1.02 \%$ of all trials. Assumptions of normality and homogeneity were checked by visual inspection of the histogram, qq-plot as well as a Shapiro-Wilk and Kolmogorov-Smirnov test. In order to meet the assumptions a logarithmic transformation of the RT data and an arc-sine transformation of the accuracy rates were performed. Analyses were performed with and without outliers (i.e. more than two standard deviations below or above the group's mean). If and when the exclusion of the outliers changed the results, this was stated clearly. All analysis were conducted using the general statistical software package SAS, Version 9.4 of the SAS System for Windows (SAS University Edition 2013). Significance tests were conducted with a significance level of $p<.05$. Post-hoc tests were Tukey-Kramer-corrected.

First, we evaluated the overall influence of the betweensubjects variables Block Order (global-then-local vs. localthen-global) and Global Type (snake vs. shape) in two separate analyses. Both factors were manipulated as between-subjects variables. The number of participants assigned to each condition can be found in Table 2 . We selected sub-samples of the original ASD and TD groups, such that the ASD and TD sample were group-wise matched (see Tables 4, 5 in Online Appendix 1). Then two repeated-measures mixed models were applied, including Group (ASD vs. TD), one of the two between-subjects variables (either Block Order or Global Type), and the interaction (Block Order $\times$ Group, or Global Type $\times$ Group, respectively) as fixed factors in our model, and both subject and intercept as random factors. As these preceding analyses did not reveal a differential group effect of the between-subjects variable (i.e., no significant interaction between Group $\times$ Block Order nor between Group $\times$ Global Target; see Results section), the respective between-subjects factors were not included in further analyses.

Next, matched participant samples were again selected (see Table 1), and a mixed models analysis was performed. We included the following fixed factors in the full model: Group (ASD vs. TD), Target Level (Local vs. Global), Distractor condition (Baseline vs. Congruent vs. Incongruent), and all two- and three-way interactions. Subject and intercept were included as random factors. A backward selection procedure was used, taking fit statistics into account. Only results of the final model are reported.

\section{Results}

\section{Preceding Analyses of Between-Subjects Factors}

A repeated-measures mixed model analysis with Group and Block Order as between-subject variable and accuracy as dependent variable, revealed a main effect of Group, indicating that the ASD sample was outperformed by the TD group $(F(1,50)=6.83, p=.01)$. No main effect of Block $\operatorname{Order}(F(1,50)=.40, p=.53)$ nor a significant Group $\times$ Order interaction $(F(1,50)=1.17, p=.28)$ were found. A similar model with $\log \mathrm{RT}$ as dependent variable revealed a trend for Block $\operatorname{Order}(F(1,50)=3.63$, $p=.06$; after exclusion of outliers: $F(1,48)=8.00$, $p<.01$ ): across both participant groups, responses tended to be slower in the Local-then-global condition compared to the Global-then-local condition. No main effect of Group $(F(1,50)=.13, p=.72)$ nor a significant Block Order $\times$ Group interaction $(F(1,50)=.78, p=.38)$ were found.

A repeated-measures mixed model analysis with Group and Global Type as between-subject variable and accuracy as dependent variable revealed a main effect of Group $(F(1,50)=9.53, p<.005)$ and a main effect of Global Type $(F(1,50)=4.79, p=.03)$ but no significant of Group $\times$ Global Type interaction effect $(F(1,50)=.70$, $p=.41)$. The TD group was significantly more accurate than the ASD sample, and snakes were detected with a 
Fig. 1 Examples of the stimuli used in Experiment 1. Targets are encircled with a full line. Distractors are encircled with a dashed line. The local targets (top row) were constructed as one single Gabor element. The global targets were constructed from multiple Gabor elements constituting either an open curvilinear arrangement (middle row) or a closed random shape (bottom row). In the baseline condition (left column) no distractor was present in the consistent distractor condition (middle column) the distractor was located in the same half of the screen as the target. In the inconsistent distractor condition (right column) the distractor was located in the opposite half of the screen as the target. All stimuli were created with the Grouping Elements Rendering Toolbox (GERT; Demeyer and Machilsen 2012)
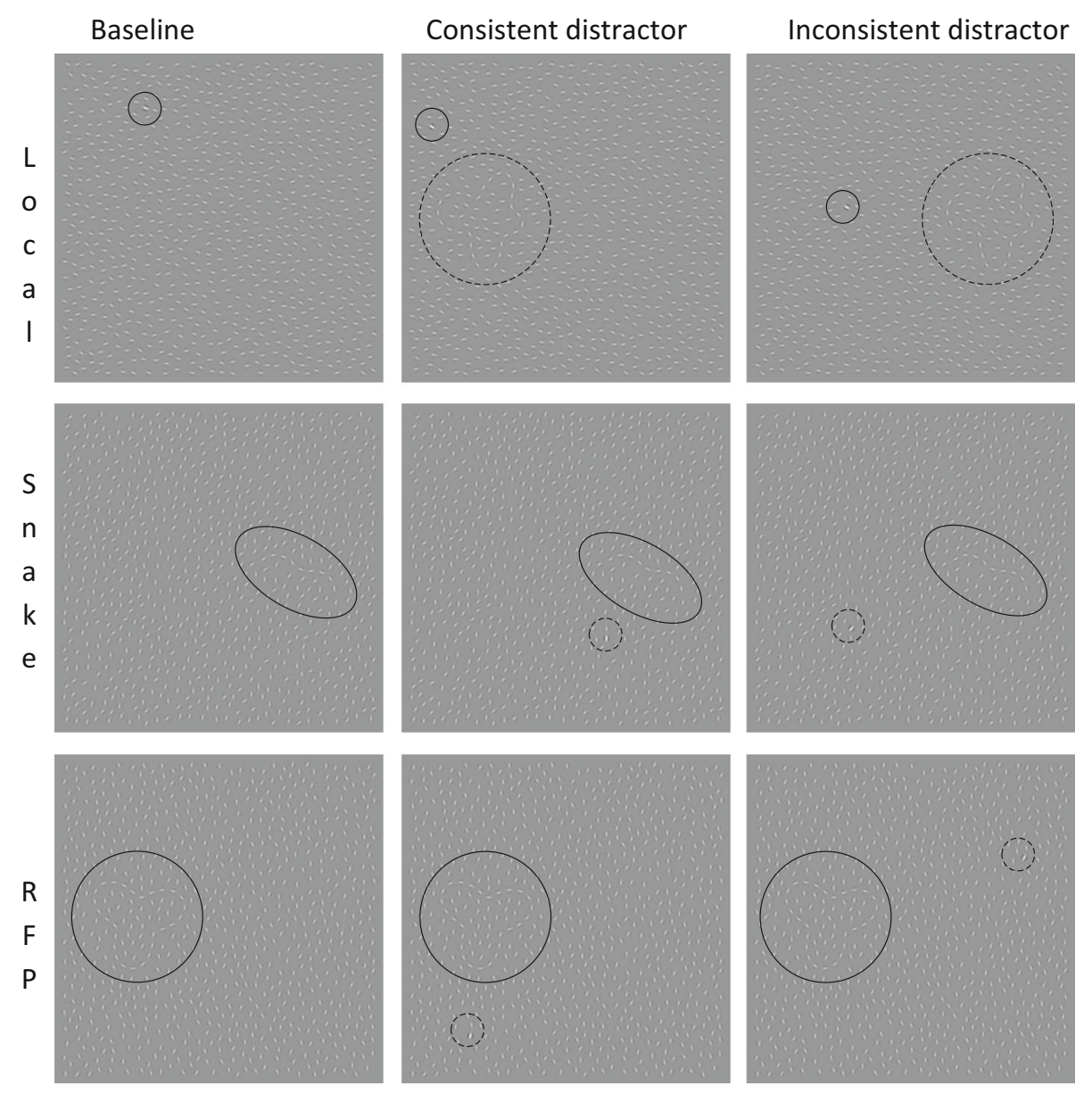

higher accuracy than closed shapes. However, no evidence for a differential effect of Global Type across both participant groups was found. A similar model with $\operatorname{logRT}$ as dependent variable, however, revealed no main effect of Group $(F(1,50)=.22, p=.64)$, Global Type $(F(1$, $50)=.31, p=.58)$, nor Group $\times$ Global Type interaction $(F(1,50)=.30, p=.58)$. Neither of both groups was faster than the other, nor were they faster for closed shapes compared to snakes, or otherwise.

\section{Main Analyses of the Within-Subject Factors}

Following our strategy as described in Data Analysis, Order and Global Type were not incorporated in further analyses.

A repeated-measures mixed model analysis with Group as between-subject variable, Target Level and Distractor Condition as within-subject variables, and accuracy as dependent variable, revealed a main effect of Target Level $(F(1,53)=7.05, p=.01)$ and a main effect of Group $(F(1,52)=7.32, p<.01)$. Overall, participants were more accurate for local targets than for global targets, and the TD group proved more accurate than the ASD group. No main effect of Distractor condition $(F(2,106)=1.89, p=.16)$, or no significant interaction effects were revealed $($ Group $\times$ Target Level: $F(1,52)=.15, p=.70 ;$ Group $\times$ Distractor condition: $F(2,104)=1.03, \quad p=.36)$; Group $\times$ Distractor condition $\times$ Target Level: $F(2$, 104) $=1.53, p=.22$ ).

A similar model, with $\log \mathrm{RT}$ as dependent variable revealed a main effect of Target Level (F(1, $52)=3762.56, p<.0001)$, a main effect of Distractor condition $(F(2,106)=14.46, p<.0001)$ and a significant interaction between Group $\times$ Target Level $(F(1$, $52)=7.09, p=.01)$ and Distractor condition $\times$ Target Level $(F(2,106)=12.70, p<.0001)$. There was no main effect of $\operatorname{Group}(F(1,52)=.18, p=.68)$, nor significant interaction effects between Group $\times$ Distractor condition $(F(2,104)=1.16, p=.32)$, or Group $\times$ Distractor condition $\times$ Target Level $(F(2,104)=.10, p=.91)$. The main effect of Target Level revealed that globally defined targets were generally found faster than locally defined targets $(F(1,52)=3762.56, p<.0001)$, whereas the main effect of Distractor Condition revealed that baseline trials without distractor were detected faster than incongruent or congruent distractor trials $(F(2,106)=14.46, p<.0001)$. 
However, the significant Distractor condition $\times$ Target Level interaction indicated that this speed advantage for baseline trials was only present for the detection of locally defined targets in the presence of a globally defined distractor $(F(2,106)=26.62, p<.0001)$ and not for a globally defined target in the presence of a locally defined distractor $(F(2,106)=.56, p=.5716)$, suggesting that the local distractors had a negligible effect on the detection of global targets, across both participant groups. No main effect of Group was found $(F(1,52)=.18, p=.6752)$, however, a Group $\times$ Target Level interaction was revealed $(F(1,52)=7.09, p=.0103)$. Post-hoc tests revealed that both groups were faster on global targets compared to local targets. The effect of Target Level proved significant within each group. Visual exploration of the data (see Fig. 3) suggests that this effect of Target Level was more accentuated for the ASD group, compared to the TD group. However, no additional effect of Group was revealed for either types of targets $(p>.35)$.

\section{Experiment 2}

\section{Methods}

\section{Participants}

The research protocol was administered 8-to-14-year old Dutch-children who fulfilled the same inclusion and exclusion criteria as described in Experiment 1, and were recruited in the same manner. Demographic details of the ASD and TD group can be found in Table 3. Note that there is no overlap in participants between Experiment 1 and Experiment 2. However, most of the participants of Experiment 2, did take part in a previous, though unrelated study (Van der Hallen et al. 2015b).

The experimental group consisted of 26 children with a formal clinical diagnosis of ASD. ASD diagnoses were reconfirmed within the research protocol using the Dutch version of the ADOS-2 conducted by a trained clinical psychologist (Gotham et al. 2006; Dutch version: de Bildt et al. 2009) in 25 of the 26 children. The comparison group consisted of 27 typically developing children. Participants with and without ASD were group-wise matched based on intelligence, age and gender-ratio.

Intellectual abilities for all participants were estimated by administering an abbreviated version (Sattler 2001) of the WISC-III-NL (Wechsler 1992). ASD symptomatology was evaluated using the SRS (Roeyers et al. 2011).

\section{Procedure}

The study was approved by the ethical committee of the university hospital and was incorporated within a larger series of studies on visual perception in ASD. The order of the different experiments was randomized, with long breaks in-between experiments. Participation was rewarded with a small present and parents received a mileage allowance, when applicable.

As in Experiment 1, all participants were individually tested in a quiet and darkened room. Participants were seated approximately $57 \mathrm{~cm}$ from a 17 -in. computer screen (pixel resolution 1024 by 768, 16-bit color, $75 \mathrm{~Hz}$ refresh rate). They were presented with a Gaborized search task and instructed to indicate whether the predefined target was situated on the left or right side of the screen, by pressing one of two buttons on a two-button response-box. Participants were encouraged to respond as fast and accurately as possible.

The experiment consisted of three smaller blocks with an explicitly defined target, and one large block without explicit instructions. First, all three stimulus conditions (i.e., local, open and closed targets) were presented in separate 'explicit' blocks of 80 trials each. The presentation order of these trial types was counterbalanced between participants. Thereafter, participants were presented with a fourth 'implicit' block of 120 trials. Here, all three target types $(3 \times 40$ trials each) were presented intermingled, during the practice trials as well as when completing the test trials. In each trial, only one target stimulus was present, appearing on the left or right side of the screen. At the start of each trial, a two-second fixation cross was followed

Table 3 Experiment 2: participant characteristics

\begin{tabular}{|c|c|c|c|c|c|}
\hline & \multicolumn{2}{|c|}{ ASD group ( $n=26: 19$ boys, 7 girls) } & \multicolumn{2}{|c|}{ TD group ( $n=27: 17$ boys, 10 girls $)$} & \multirow{2}{*}{$\begin{array}{l}\text { Two-sided } t \text { test } \\
p \text { value }\end{array}$} \\
\hline & Mean & SD & $\overline{\text { Mean }}$ & SD & \\
\hline Age (years) & 10 & 2 & 10 & 1 & .79 \\
\hline Verbal IQ & 98 & 14 & 103 & 12 & .13 \\
\hline Performance IQ & 103 & 12 & 103 & 14 & .95 \\
\hline SRS & 90 & 16 & 48 & 6 & $<.0001$ \\
\hline ADOS & 5.96 & 1.71 & N/A & N/A & N/A \\
\hline
\end{tabular}


by the appearance of the test stimulus. Immediate feedback was provided, both in the practice and the test trials, as each response was followed by a green (correct) or red (incorrect) screen. If participants did not respond within the eight-second time limit, a red screen was provided and the next trial was presented. All participants completed an extensive step-by-step practice protocol with 8 practice trials before commencing with each block of test trials. Stimuli were presented and responses were recorded within the E-prime environment (E-prime version 1.1.41. PST Inc.).

\section{Stimuli}

Stimuli consisted of $25.71^{\circ}$ by $17.53^{\circ}$ displays of randomly oriented Gabor elements containing a target either on the left or right side of the display. As in Experiment 1, all stimuli were created with the GERT toolbox (Demeyer and Machilsen 2012).

For the local targets, one single Gabor element was oriented differently from the orientation of the surrounding background elements, who all had (more or less) the same randomly chosen orientation (see below). Differences in the difficulty level (or complexity) of detecting the local target were achieved by (1) manipulating the degree of orientation deviation, namely either $50^{\circ}$ ('simple') or $35^{\circ}$ ('complex') relative to the orientation of the background Gabors, and (2) by presenting the background elements with or without orientation jitter (orientations varying between $+12^{\circ}$ and $-12^{\circ}$ ). The open (global) targets were constructed from multiple aligned Gabor elements constituting an open contour. The complexity of the open contour was manipulated by employing a clearly-defined straight line ('simple') or a less clearly-defined curved line ('complex'). The elements of the contour were either presented with or without orientation jitter (orientations varying between $+30^{\circ}$ and $-30^{\circ}$ ). Background elements were presented in random orientation. The closed (global) targets were constructed from multiple aligned Gabor elements constituting a closed contour. The complexity of the closed contour was manipulated by variation of the number and amplitude of the sinusoidal frequencies of the RFPs, yielding shapes with less ('simple') or more ('complex') curvature variation. The elements of the contour were either presented with or without orientation jitter (orientations varying between $+30^{\circ}$ and $-30^{\circ}$ ). Background elements were presented in random orientation. Examples of the stimuli can be found in Fig. 2.

\section{Data-Analysis}

RTs and mean accuracy were analyzed. Only RTs (expressed in milliseconds) of correctly responded trials were analyzed, and RTs smaller than $100 \mathrm{~ms}$ or over $10,000 \mathrm{~ms}$ were excluded from further analysis, resulting in the exclusion of $.98 \%$ of all trials. Assumptions of normality and homogeneity were checked by means of a visual inspection of the histogram, qq-plot as well as a ShapiroWilk and Kolmogorov-Smirnov test. As in Experiment 1, a logarithmical transformation for the RT data and an arcsine transformation for the accuracy rates were performed. Analyses were performed with and without outliers (i.e. more than two standard deviations below or above the group's mean). If and when the exclusion of the outliers changed the results, this was stated clearly. All analysis were conducted using the general statistical software package SAS, Version 9.4 of the SAS System for Windows (SAS University Edition 2013). Significance tests were conducted with a significance level of $p<.05$. Post-hoc tests were Tukey-Kramer-corrected (Fig. 3).

Our research design includes Group as between-subject variable, and Instruction, Target Type, Noise and Complexity as within-subject variables. The variable Instruction refers to the two types of task instruction: an explicit task instruction (three shorter blocks of trials, each with its own predefined target type) or an implicit task instruction (one longer block with all target types intermingled). The Target Type refers to the three types of target stimuli, namely local, open global and closed global search targets. Noise refers to the absence or presence of orientation noise (or jitter) on the background elements (in case of local targets) or the contour elements (in case of open and closed global targets). Complexity refers to the complexity level of the target stimulus displayed, which was either a 'simple' or a 'complex' version of the search target (for the precise manipulations, which differed for each target type, see Stimuli section). None of the two-way interactions with Noise and Complexity yielded particularly interesting effects. In the interest of readability, these results are included as Supplementary Material (see Online Appendix 2 ), rather than reported and discussed in the main text. Subject and intercept were included in the analysis as random factors.

\section{Results}

A repeated-measures mixed model analysis with accuracy as dependent variable, Group as between-subject variable, and Instruction, Target Type, Noise and Complexity as within-subject variables, revealed a main effect of Instruction $(F(1,51)=18.87, p<.0001)$, Target Type $(F(2$, $102)=45.33, \quad p<.0001)$, Noise $(F(1,51)=369.12$, $p<.0001)$ and Complexity $(F(1, \quad 51)=735.50$, $p<.0001)$, as well as a two-way interaction effect of Group $\times$ Instruction $(F(1,51)=6.02, p=.0176)$ and Target Type $\times$ Instruction $(F(2,103)=19.37, p<.0001)$. 
Fig. 2 Examples of the stimuli used in Experiment 2. Targets are encircled with a full line.

The local targets (top row) were constructed as one single Gabor element. The global targets were constructed from multiple Gabor elements constituting either an open contour (middle row) or a closed random shape (bottom row). Of each type of stimuli, both a more simple (left column) and more difficult (right column) version was created. In addition, all stimuli were presented with or without orientation jitter (here shown without orientation jitter). All stimuli were created with the Grouping Elements Rendering Toolbox (GERT; Demeyer and Machilsen 2012)
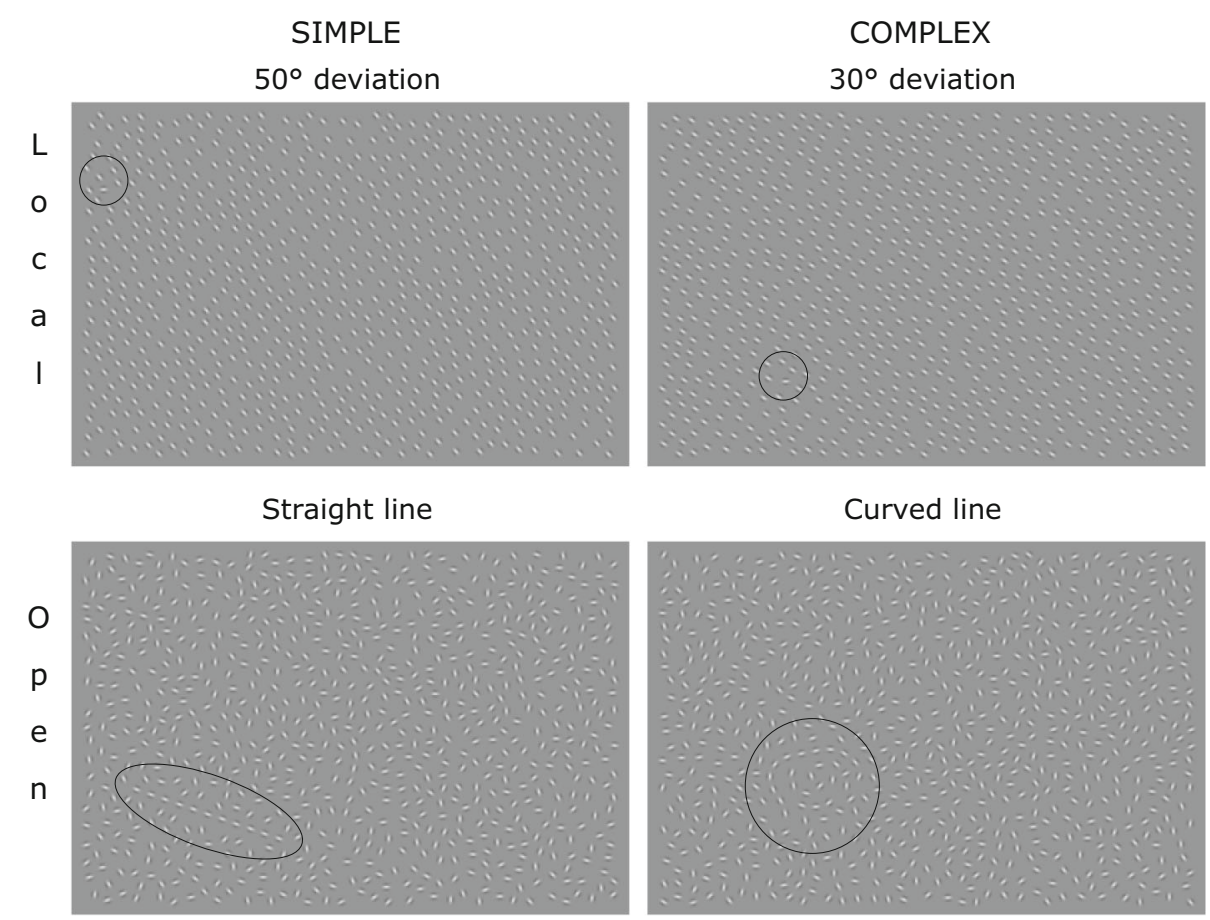

Simple shape
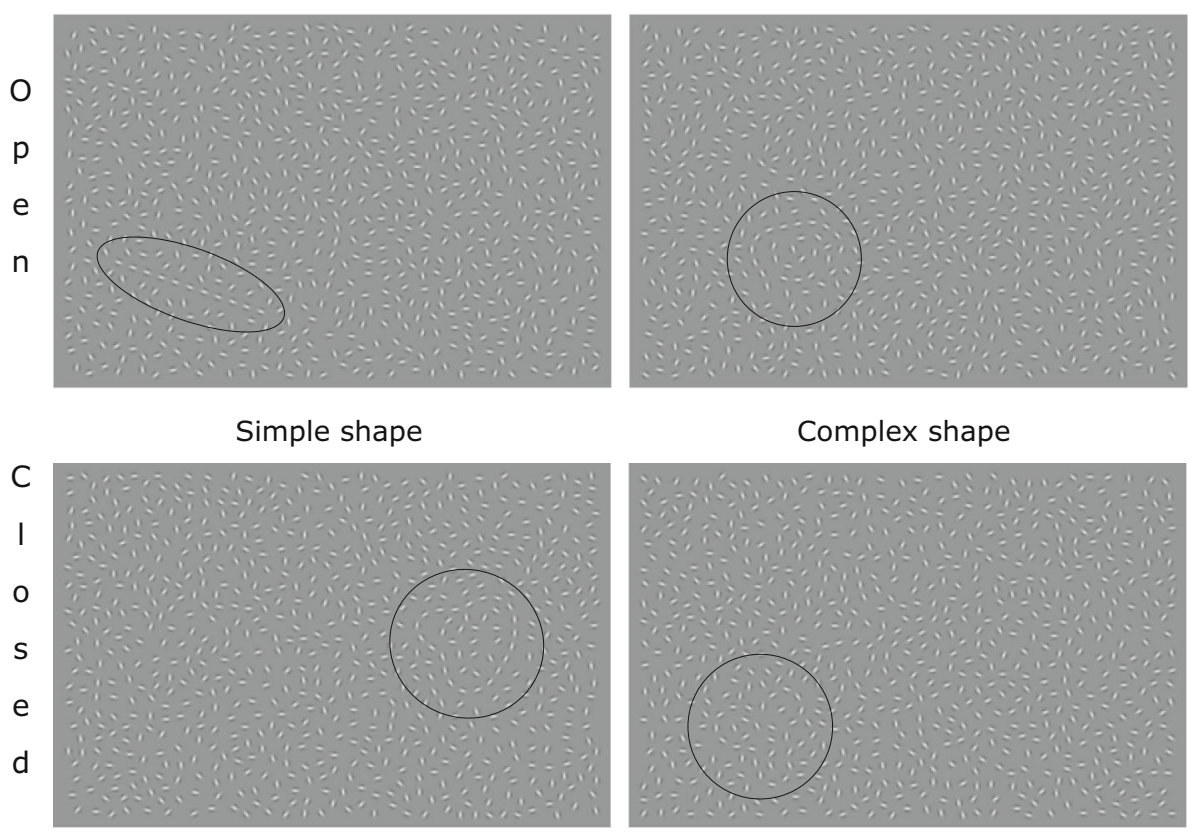

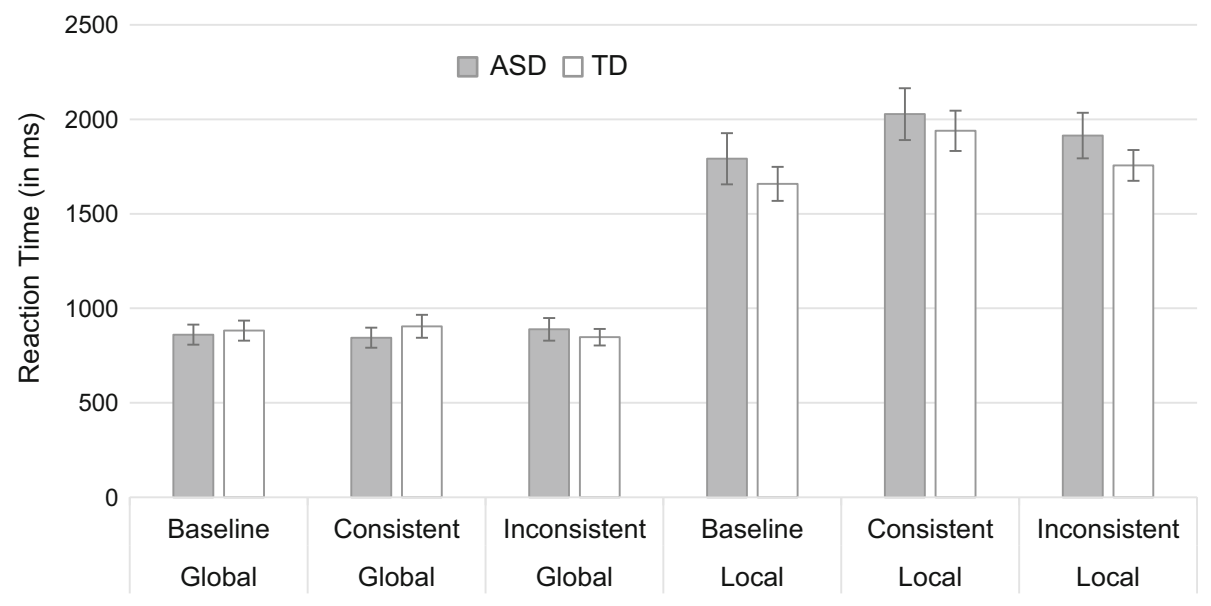

Fig. 3 Mean reaction times of Experiment 1 for the ASD and TD group, for both target levels (Local vs. Global) and all three distractor conditions (Baseline vs. Congruent vs. Incongruent). A repeatedmeasures mixed model analysis with $\operatorname{logRT}$ as dependent variable revealed a main effect of Target Level $(\mathrm{F}(1,52)=3762.56$, $p<.0001)$, a main effect of Distractor condition $(\mathrm{F}(2$, $106)=14.46, p<.0001)$ and a significant interaction between Group $\times$ Target Level $(\mathrm{F}(1,52)=7.09, p=.01)$ and Distractor condition $\times$ Target Level $(\mathrm{F}(2,106)=12.70, p<.0001)$. Error bars represent standard error of the mean (SEM) 
None of the three-way interactions proved significant $(p>.12)$. The main effect of Instruction revealed that, for both ASD and TD group, participants were more accurate on the second task with implicit task instruction $(M=.92$, $S D=.13)$, compared to the first task with explicit task instruction $(M=.91, S D=.12)$. However, the significant interaction between Group $\times$ Instruction (see Fig. 4) revealed that, whereas the typically developing group was more accurate in the implicit condition compared to the explicit condition $(t(51)=-4.84, p<.0001)$, the ASD sample performed as accurately in the implicit condition as in the explicit condition $(t(51)=-1.33, p=.1904)$. Note that this effect cannot be due to a differential learning effect in the explicit condition, as additional analyses show a main effect of learning in the explicit condition $(F(1$, $51)=6.00, p=.02$ ), but no interaction effects (see Online Appendix 3). The main effect of Target Type revealed that, for both ASD and TD group, participants were most accurate in finding open targets $(M=.94, S D=.10)$, second most accurate for closed targets $(M=.91$, $S D=.12)$ and least accurate for local targets $(M=.89$, $S D=.14)$ (post hoc comparisons $p<.005)$. The significant interaction between Target Type $\times$ Instruction revealed that the most difficult targets were the local ones in the explicit condition and the closed ones in the implicit condition, while the open targets were the easiest in both conditions. The main effect of Noise revealed that, for both participants groups, target detection was more accurate in the absence of noise $(M=.96, S D=.08)$ than when orientation noise was present $(M=.87, S D=.14)$. The main effect of Complexity revealed that, for both participant groups, simple targets were found more accurately $(M=.97, S D=.05)$ than complex targets $(M=.85$, $S D=.15)$.
A similar repeated-measures mixed model analysis with $\operatorname{logRT}$ as dependent variable revealed similar effects: a main effect of Instruction $(F(1,51)=92.64, p<.0001)$, Target Type $(F(2,102)=364.56, p<.0001)$, Noise $(F(1$, $51)=359.99, \quad p<.0001) \quad$ and Complexity $(F(1$, $51)=1192.78, p<.0001)$, as well as two-way interaction effects of Group $\times$ Instruction $(F(1,51)=11.66$, $p=.0013)$ and Target Type $\times$ Instruction $\quad(F(2$, $103)=8.89, p=.0003$ ). None of the three-way interactions proved significant $(p>.12)$. The main effect of $I n$ struction revealed that, for both ASD and TD group, participants were faster on the second task with implicit task instruction $(M=1181, S D=404.92)$, compared to the first task with explicit task instruction $(M=1311$, $S D=476.17)$. However, the significant Group $\times I n$ struction interaction revealed that this effect was stronger for the TD group than for the ASD group (see Fig. 5). Note, again, that this effect cannot be due to a differential learning effect in the explicit condition, as additional analyses show a main effect of learning in the explicit condition $(F(1,51)=23.70, p<.0001)$, but no interaction effects (see Online Appendix 3). The main effect of Target Type revealed that both the ASD and TD children were fastest finding the open targets $(M=1078, S D=352.42)$, than the closed targets $(M=1179, S D=421.19)$, and slowest finding the local targets $(M=1478, S D=459.87)$ (all post hoc comparisons $p<.0001$ ). The significant interaction between Target Type $\times$ Instruction indicated that, while all three target types were detected faster in the implicit condition, this improvement in RT was the largest for the local targets. The main effect of Noise revealed that, for both participants groups, detection speed was faster in the absence of noise $(M=1129, S D=393)$ than when orientation noise was present $(M=1362, S D=467)$. The

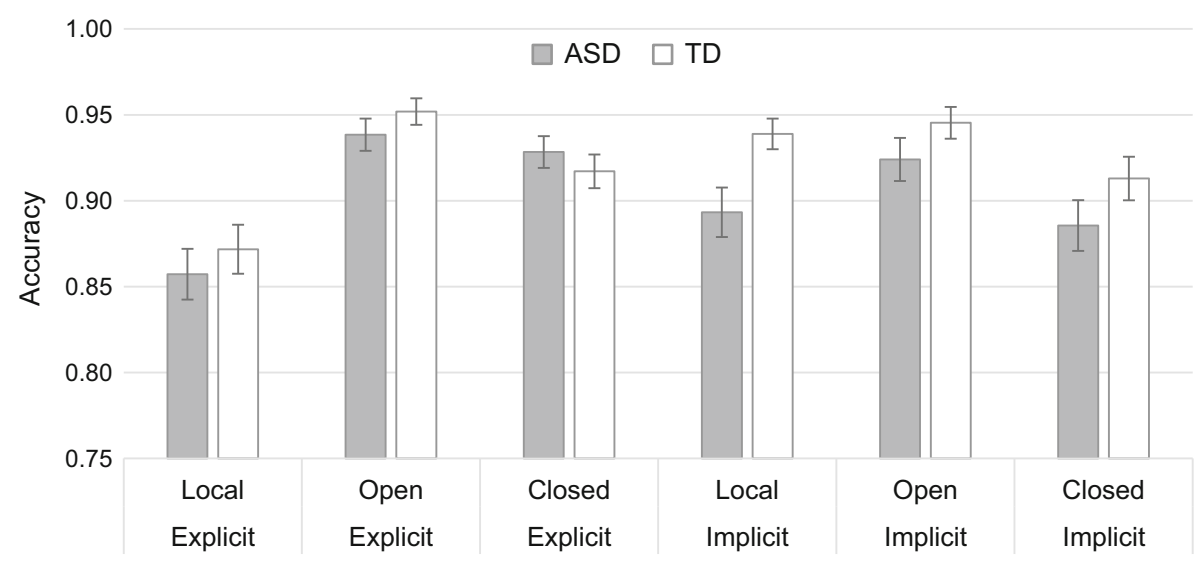

Fig. 4 Mean accuracy scores of Experiment 2 for the ASD and TD group, for both instruction levels (Explicit vs. Implicit) and all three target types (Local vs. Open vs. Closed). A repeated-measures mixedmodel analysis with accuracy as dependent variable revealed a main effect of Instruction $(\mathrm{F}(1,51)=18.87, p<.0001)$, Target Type $(\mathrm{F}(2$,
$102)=45.33, p<.0001)$, Noise $(\mathrm{F}(1,51)=369.12, p<.0001)$ and Complexity $(\mathrm{F}(1,51)=735.50, p<.0001)$, as well as a two-way interaction effect of Group $\times$ Instruction $(\mathrm{F}(1,51)=6.02$, $p=.0176)$ and Target Type $\times$ Instruction $(\mathrm{F}(2,103)=19.37$, $p<.0001)$. Error bars represent SEM 


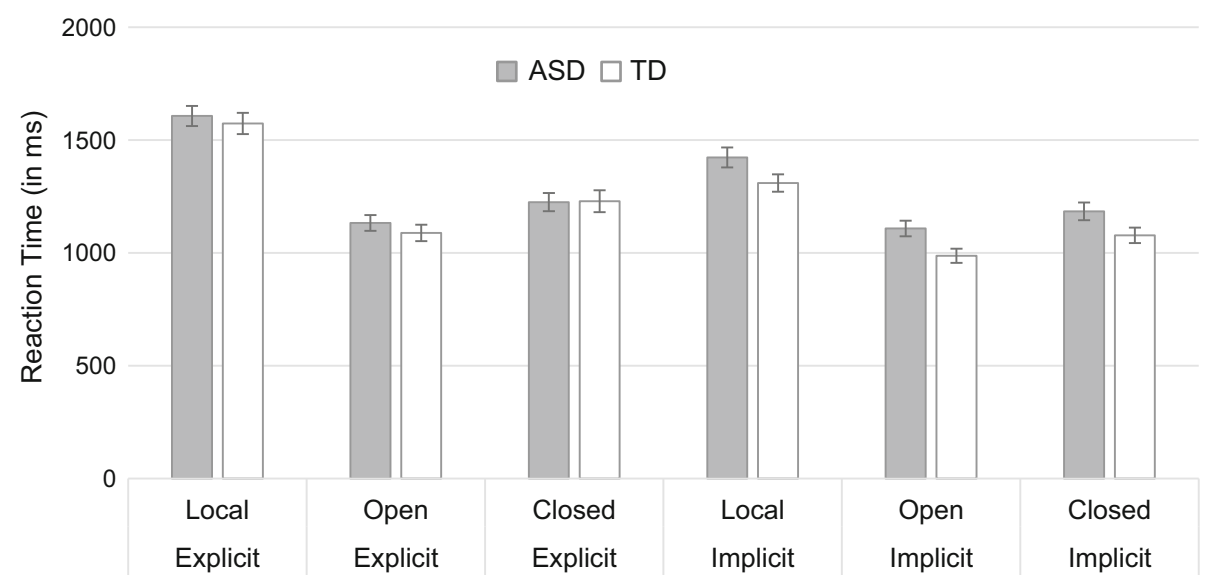

Fig. 5 Mean reaction times of Experiment 2 for the ASD and TD group, for both instruction levels (Explicit vs. Implicit) and all three target types (Local vs. Open vs. Closed). A repeated-measures mixedmodel analysis with $\log \mathrm{RT}$ as dependent variable revealed a main effect of Instruction $(\mathrm{F}(1,51)=92.64, p<.0001)$, Target Type $(\mathrm{F}(2$, $102)=364.56, p<.0001)$, Noise $(\mathrm{F}(1,51)=359.99, p<.0001)$

main effect of Complexity revealed that, for both participant groups, simple targets were found faster $(M=1023$, $S D=271.06)$ compared to complex targets $(M=1467$, $S D=477.12)$.

\section{Discussion}

The current study employed a Gaborized visual search paradigm to assess the impact of task characteristics in individuals with and without ASD. All participant groups were group-wise matched for age, gender and FSIQ. Experiment 1 employed visual search to investigate the impact of distractor interference. Participants were instructed to indicate whether a predefined (local or global) target was situated on the left or right side of the screen, while ignoring a possible (local or global) distractor. Although some interesting effects were revealed, the predicted three-way interaction of Group by Distractor Condition by Target Level was not revealed: both groups proved equally sensitive to the absence or presence of a distractor, regardless of the type of target or type of distractor. Experiment 2 employed a search paradigm to investigate the influence of implicit versus explicit task directions on local-global visual processing. Participants were instructed to indicate whether a predefined (local, open global or closed global) target was situated on the left or right side of the screen, both under implicit and explicit task directions. In line with our expectations, results showed a differential effect of task instruction for ASD compared to TD; while both groups performed equally well and Complexity $(\mathrm{F}(1,51)=1192.78, p<.0001)$, as well as two-way interaction effects of Group $\times$ Instruction $(\mathrm{F}(1,51)=11.66$, $p=.0013)$ and Target Type $\times$ Instruction $(\mathrm{F}(2,103)=8.89$, $p=.0003)$. None of the three-way interactions proved significant $(p>.12)$. Error bars represent SEM

when given an explicit task instruction, the TD group outperformed the ASD group when given an implicit task instruction. However, no Group by Target Type interaction effect was found.

\section{Distractor Interference}

Although distractor tasks are not commonly used in ASD inhibition literature, the available evidence suggests increased interference for individuals with ASD, compared to TD participants (Adams and Jarrold 2012). The current study, however, found a similar degree of distractor interference for both groups. In addition, sensitivity to the distractors was not modulated differently by local versus global target types for both groups. As previously explained, most of the previous studies that revealed impaired distractor interference in ASD used a flanker task (Adams and Jarrold 2012; Burack 1994; Christ et al. 2007, 2011; Remington et al. 2009). In these flanker tasks, participants are usually aware of the precise location of the distractor(s), i.e., flanking the target, while unaware of the particular nature (congruent or incongruent) of the distractor(s). The same holds for the location of the distractors elements used in classical Navon paradigms. In the current Gaborized search paradigm, however, participants were unaware of the location of the distractor, probably making it more difficult to ignore and thereby increasing the overall task demands. In addition, targets and distractors in the current study were two separate stimuli which were embedded in a field of Gabor patches (thus embedded within a larger stimulus), while in most other designs target 
and distractor elements are combined within one distinct core stimulus (as is the case with the two-level hierarchical Navon stimuli). In line with Lavie's (1995) load-account, a study by Wilson et al. (2008) has suggested that task load or task demands are the main determinant of the extent to which distractors are processed. With their study, they have provided solid evidence that task load and distractor interference correlate negatively: As Wilson and colleagues increased the task load, distractor interference decreased, regardless of the location of the distractor (fixation or periphery), the distinctiveness of the distractor, or the absolute level of interference. In the current study, task load was not only increased by the fact that participants were unware of the distractor-location, but also due to the fact that both the target and distractor were embedded in a field of Gabor patches, which, evidently, functioned as distractors or camouflage-elements, themselves.

\section{Implicit Versus Explicit Task Instructions}

Whereas studies on implicit versus explicit task directions are fairly common in relation to executive functioning in ASD (for example, see Van Eylen et al. 2015), not many studies have investigated the impact of task directions in relation to local-global visual processing. Koldewyn et al. (2013) were the first to hint at the importance of task instruction for ASD vision research. With their research, Koldewyn et al. indicated that children with ASD have intact global information processing abilities, yet are less inclined to attend to and report global information. Here, we show a differential effect of task instruction for participants with and without ASD: While both groups performed equally well when explicitly instructed to search for a certain target type, the TD group outperformed the ASD group under implicit task instruction (i.e., when the target type was not specified). One could argue that the effect of task instruction merely reflects a difference in learning curve, as the explicit task condition always preceded the implicit task condition. Such an interpretation of the data would suggest that while the TD group improved over time (as their performance increased from the explicit to the implicit condition) such learning effect is lacking for the ASD group (as their performance did not improve from the implicit to the explicit condition). However, several previous studies found (explicit) learning to be intact in ASD (e.g., Brown et al. 2010; Nemeth et al. 2010). A direct test of differential learning for our data revealed a significant learning effect in the explicit task, both in terms of accuracy and RT performance, however, this learning effect was similar for both groups (for more details, see Online Appendix 3). Therefore, it seems safe to argue that the ASD group in our study did not suffer from a learning deficit, but that the lack of improvement on their behalf, is due to the implicit nature of the second condition. The participants with ASD did learn, as did their TD counterparts, but their performance in the implicit condition got hindered by the randomized presentation of different types of targets, which left the ASD participants (literally) "clueless" as to what target to look for. Impairments of behavioral and cognitive flexibility might have hindered the ASD group to quickly deduct the correct type of target and respond adequately, and to switch from one trial to the next. Behavioral and cognitive inflexibility is manifested by an insistence of sameness and resistance of change, making it more difficult to adapt and respond flexibly to new situations (Green et al. 2007). In the current study, this relates to the situation where the task instruction is given implicitly and flexibility is required to adapt to the task where target stimuli are presented in a randomized manner.

The Task Support hypothesis has also yielded some interesting findings related to memory research that correspond nicely with our findings. In an elegant task by Bowler et al. (1997) participants were asked to perform two distinct memory tasks. In a first task, comparable to our implicit task, participants were given a general instruction to study word lists and to remember as many of the lists as possible. In a second task, comparable to our explicit task, participants were asked to remember similar lists of words but the encoding and retrieval strategies of participants were tightly constrained by the demands of the task. In other words: while the first task was unconstrained in terms of memory strategies (or lacked support in terms of memory strategies), the second task provide clear constraints or support with regard to which memory strategies to employ. Their data, and the results of several other researchers since (e.g., Bowler et al. 2004; Gaigg et al. 2008) showed that participants with ASD, compared to TD individuals, suffered from memory impairment under the unconstrained conditions, while their performance was unimpaired performance when task constraints were strong. Although these results pertain to memory, and not visual processing, the relationship between performance of the ASD group and the particular task conditions entails an interesting parallel with our findings.

\section{Details and Wholes}

Differences in local-global visual processing between individuals with ASD and TD individuals have long been the subject of discussion (for a review, see Simmons et al. 2009; for a meta-analysis, see Van der Hallen et al. 2015b). While in the earlier years, atypical visual processing in ASD was viewed in terms of inabilities and deficits, in the more recent years, atypical visual processing in ASD is discussed in terms of difference in inclination or automatic modes. Whereas TD individuals are known to be "global 
processers", individuals with ASD are thought to be more automatically drawn to more local, detailed elements in visual processing. In the current study, we employed a range of targets, comprising local targets (composed of merely one Gabor patch), open global targets (lines or snakes) as well as closed global targets (shapes or RFPs), in order to leave room for two- or three-way interaction effects between both groups, the types of targets used, and the particular task manipulations. However, both groups proved fastest for open global targets (snakes or lines), second fastest for closed, global targets, and slowest for local targets. Hence, the global targets were the easiest, both for the ASD group and TD group. No evidence was found of a local processing bias for ASD. On the contrary, the Group by Target Level interaction in Experiment 1 suggests a disadvantage for ASD on the local, more difficult, targets, compared to the global targets. A recent study by Jachim et al. (2015) investigated contour integration for open and closed contours in participants with ASD and matched controls. In support of altered integration, their results indicated a detection advantage for closed contours, present in both the TD and ASD group, though smaller for the ASD group. Again, in the current study, no such effect was found, although the advantage for closed contours itself, was replicated.

While the lack of a group by target type interaction effect was perhaps to be expected for Experiment 1 and the explicit conditions of Experiment 2, given their explicit, structured nature, it comes as more of a surprise that such an interaction effect is not present for the implicit condition of Experiment 2. Here, there is far more room for differences in spontaneous, automatic processing to result in time or accuracy differences between both participant groups. However, no such effects were revealed.

\section{In Sum}

In the current study, two visual search tasks were employed to assess the impact of task characteristics in individuals with and without ASD. Despite the recent claims that, across development and a broad range of symptom severity, individuals with ASD tend to outperform controls on visual search task (Kaldy et al. 2013), neither of our experiments found improved performance for the ASD group. In addition, neither of these experiments found strong differences in sensitivity to local versus global levels of visual stimuli. Differences in sensitivity to the particular task conditions, however, were pronounced. Taken together, these data underline the importance of task factors in the study of local-global visual processing in ASD.
Acknowledgments First of all, the authors would like to thank all participants and their families for their time and contribution to this research, as well as the participating schools for allowing us into their classrooms. Secondly, we would like to thank Bart Machilsen and Maarten Demeyer for their help with stimulus generation and the GERT toolbox. In addition, we would like to thank Birgitt Haesen for the experimental designs and for her assistance in the data collection. Last but not least, we would like to thank our master students Nele Berghmans, Sanne Drees, Sophie Duchesne, Helen Goovaerts, Ellen Janssen, Nele Soors, Astrid Van Der Most, and Lotte van Esch, for their collaboration on this project and/or help with the data collection. This research was funded by a Methusalem grant awarded to Johan Wagemans by the Flemish Government (METH/08/02 and METH/ 14/02), two postdoctoral fellowships of the Research Foundation Flanders awarded to Bart Boets and Kris Evers, and two individual grants from the Marguerite Marie Delacroix Support Fund awarded to Kris Evers (GV/B-141) and Birgitt Haesen (GV/B-119).

Author Contributions RVDH, KE, BB, JS, IN and JW were involved in the study conception and design. RVDH and KE were involved in the acquisition of the data. RVDH, KE, BB and JW were involved in the analysis and interpretation of data. RVDH and KE were involved in drafting the manuscript. RVDH, KE, BB, JS, IN and JW were involved in the critical revision of the manuscript.

\section{Compliance with Ethical Standards}

Conflict of interest All authors declare to have no conflict of interest.

\section{References}

Adams, N. C., \& Jarrold, C. (2012). Inhibition in autism: Children with autism have difficulty inhibiting irrelevant distractors but not prepotent responses. Journal of Autism and Developmental Disorders, 42(6), 1052-1063. doi:10.1007/s10803-011-1345-3.

American Psychiatric Association. (2000). Diagnostic and statistical manual of mental disorders DSM-IV-TR fourth edition (4th ed.). Arlington County: American Psychiatric Publishing Inc.

Behrmann, M., Thomas, C., \& Humphreys, K. (2006). Seeing it differently: Visual processing in autism. Trends in Cognitive Sciences, 10(6), 258-264. doi:10.1016/j.tics.2006.05.001.

Bowler, D. M., Gardiner, J. M., \& Berthollier, N. (2004). Source memory in adolescents and adults with Asperger's syndrome. Journal of Autism and Developmental Disorders, 34(5), 533-542.

Bowler, D. M., Matthews, N. J., \& Gardiner, J. M. (1997). Asperger's syndrome and memory: Similarity to autism but not amnesia. Neuropsychologia, 35(1), 65-70.

Brown, J., Aczel, B., Jiménez, L., Kaufman, S. B., \& Grant, K. P. (2010). Intact implicit learning in autism spectrum conditions. Quarterly Journal of Experimental Psychology, 63(9), 1789-1812. doi:10.1080/17470210903536910.

Burack, J. A. (1994). Selective attention deficits in persons with autism: Preliminary evidence of an inefficient attentional lens. Journal of Abnormal Psychology, 103(3), 535-543. doi:10.1037/ 0021-843X.103.3.535.

Christ, S. E., Holt, D. D., White, D. A., \& Green, L. (2007). Inhibitory control in children with autism spectrum disorder. Journal of Autism and Developmental Disorders, 37(6), 1155-1165. doi:10. 1007/s10803-006-0259-y.

Christ, S. E., Kester, L. E., Bodner, K. E., \& Miles, J. H. (2011). Evidence for selective inhibitory impairment in individuals with 
autism spectrum disorder. Neuropsychology, 25(6), 690-701. doi:10.1037/a0024256.

de Bildt, A., Sytema, S., van Lang, N. D. J., Minderaa, R. B., van Engeland, H., \& de Jonge, M. V. (2009). Evaluation of the ADOS revised algorithm: The applicability in 558 Dutch children and adolescents. Journal of Autism and Developmental Disorders, 39(9), 1350-1358. doi:10.1007/s10803-009-0749-9.

Demeyer, M., \& Machilsen, B. (2012). The construction of perceptual grouping displays using GERT. Behavior Research Methods, 44(2), 439-446. doi:10.3758/s13428-011-0167-8.

Eckstein, M. P. (2011). Visual search: A retrospective. Journal of Vision, 11(5), 14. doi:10.1167/11.5.14.

Eriksen, B. A., \& Eriksen, C. W. (1974). Effects of noise letters upon the identification of a target letter in a nonsearch task. Perception and Psychophysics, 16(1), 143-149. doi:10.3758/BF03203267.

Gaigg, S. B., Gardiner, J. M., \& Bowler, D. M. (2008). Free recall in autism spectrum disorder: The role of relational and itemspecific encoding. Neuropsychologia, 46(4), 983-992. doi:10. 1016/j.neuropsychologia.2007.11.011.

Gotham, K., Risi, S., Pickles, A., \& Lord, C. (2006). The autism diagnostic observation schedule: Revised algorithms for improved diagnostic validity. Journal of Autism and Developmental Disorders, 37(4), 613-627. doi:10.1007/s10803-0060280-1.

Green, V. A., Sigafoos, J., O'Reilly, M., Pituch, K. A., Didden, R., Lancioni, G. E., et al. (2007). Behavioural flexibility in individuals with autism: Theory, assessment, and intervention. In L. B. Zhao (Ed.), Autism research advances (pp. 63-77). New York: Nova Science Publishers, Inc.

Happé, F., \& Booth, R. (2008). The power of the positive: Revisiting weak coherence in autism spectrum disorders. The Quarterly Journal of Experimental Psychology, 61(1), 50-63. doi:10.1080/ 17470210701508731

Hess, R. F., May, K. A., \& Dumoulin, S. O. (2015). Contour integration: Psychophysical, neurophysiological and computational perspectives. In J. Wagemans (Ed.), The Oxford handbook of perceptual organization. Oxford, UK: Oxford University Press.

Iarocci, G., \& Armstrong, K. (2014). Age-related changes in conjunctive visual search in children with and without ASD. Autism Research: Official Journal of the International Society for Autism Research, 7(2), 229-236. doi:10.1002/aur.1359.

Jachim, S., Warren, P. A., McLoughlin, N., \& Gowen, E. (2015). Collinear facilitation and contour integration in autism: Evidence for atypical visual integration. Frontiers in Human Neuroscience. doi:10.3389/fnhum.2015.00115.

Joseph, R., Keehn, B., Connolly, C., Wolfe, J., \& Horowitz, T. (2009). Why is visual search superior in autism spectrum disorder? Developmental Science, 12(6), 1083-1096. doi:10. 1111/j.1467-7687.2009.00855.x.

Kaldy, Z., Giserman, I., Carter, A. S., \& Blaser, E. (2013). The mechanisms underlying the ASD advantage in visual search. Journal of Autism and Developmental Disorders. doi:10.1007/ s10803-013-1957-x.

Koldewyn, K., Jiang, Y. V., Weigelt, S., \& Kanwisher, N. (2013). Global/local processing in autism: Not a disability, but a disinclination. Journal of Autism and Developmental Disorders. doi:10.1007/s10803-013-1777-z.

Lavie, N. (1995). Perceptual load as a necessary condition for selective attention. Journal of Experimental Psychology: Human Perception and Performance, 21(3), 451-468. doi:10.1037// 0096-1523.21.3.451.
Machilsen, B., Wagemans, J., \& Demeyer, M. (2015). Quantifying density cues in grouping displays. Vision Research. doi:10.1016/ j.visres.2015.06.004.

Mottron, L., Dawson, M., Soulieres, I., Hubert, B., \& Burack, J. (2006). Enhanced perceptual functioning in autism: An update, and eight principles of autistic perception. Journal of Autism and Developmental Disorders, 36(1), 27-43. doi:10.1007/s10803005-0040-7.

Navon, D. (1977). Forest before trees: The precedence of global features in visual perception. Cognitive Psychology, 9(3), 353-383. doi:10.1016/0010-0285(77)90012-3.

Nemeth, D., Janacsek, K., Balogh, V., Londe, Z., Mingesz, R., Fazekas, M., et al. (2010). Learning in autism: Implicitly superb. PLoS One, 5(7), e11731. doi:10.1371/journal.pone.0011731.

O'Riordan, M., Plaisted, K., Driver, J., \& Baron-Cohen, S. (2001). Superior visual search in autism. Journal of Experimental Psychology: Human Perception and Performance, 27(3), 719-730. doi:10.1037//0096-1523.27.3.719.

Remington, A., Swettenham, J., Campbell, R., \& Coleman, M. (2009). Selective attention and perceptual load in autism spectrum disorder. Psychological Science, 20(11), 1388-1393.

Roeyers, H., Thys, M., Druart, C., De Schryver, M., \& Schittekatte, M. (2011). SRS Screeningslijst voor autismespectrumstoornissen. Amsterdam, Nederland: Hogrefe Uitgevers.

SAS University Edition. (2013). Version 9.4. Cary, NC: SAS Institute Inc.

Sattler, J. M. (2001). Assessment of children: cognitive applications, fourth edition (4th ed.). San Diego: Jerome M Sattler.

Simmons, D., Robertson, A., McKay, L., Toal, E., McAleer, P., \& Pollick, F. (2009). Vision in autism spectrum disorders. Vision Research, 49(22), 2705-2739. doi:10.1016/j.visres.2009.08.005.

Treisman, A., \& Gelade, G. (1980). A feature-integration theory of attention. Cognitive Psychology, 12(1), 97-136. doi:10.1016/ 0010-0285(80)90005-5.

Van der Hallen, R., Evers, K., Brewaeys, K., Van den Noortgate, W., \& Wagemans, J. (2015a). Global processing takes time: A metaanalysis on local-global visual processing in ASD. Psychological Bulletin, 141(3), 549-573. doi:10.1037/bu10000004.

Van der Hallen, R., Evers, K., de-Wit, L., Steyaert, J., Noens, I., \& Wagemans, J. (2015b). Multiple object tracking reveals objectbased grouping interference in children with ASD. Journal of Autism and Developmental Disorders. doi:10.1007/s10803-0152463-0.

Van Eylen, L., Boets, B., Steyaert, J., Wagemans, J., \& Noens, I. (2015). Executive functioning in autism spectrum disorders: Influence of task and sample characteristics and relation to symptom severity. European Child and Adolescent Psychiatry. doi:10.1007/s00787-015-0689-1.

Wang, Q., Cavanagh, P., \& Green, M. (1994). Familiarity and pop-out in visual search. Perception and Psychophysics, 56(5), 495-500.

Wang, L., Mottron, L., Peng, D., Berthiaume, C., \& Dawson, M. (2007). Local bias and local-to-global interference without global deficit: A robust finding in autism under various conditions of attention, exposure time, and visual angle. Cognitive Neuropsychology, 24(5), 550. doi:10.1080/ 13546800701417096

Wechsler, D. (1992). WISC-III-NLIWechsler Intelligence Scale for Children-III. New York: Psychological Corporation.

Wilson, D. E., MacLeod, C. M., \& Muroi, M. (2008). Practice in visual search produces decreased capacity demands but increased distraction. Perception and Psychophysics, 70(6), 1130-1137. 